\title{
Observer Design for Descriptor Takagi Sugeno System
}

\author{
Kilani Ilhem \\ National Engineering School of \\ Gabès , University of Gabès \\ Street Ibn El Khatab ,Zrig 6029
}

Gabès, Tunisia

\author{
Hamza Rafika \\ National Engineering School of \\ Gabès , University of Gabès \\ Street Ibn El Khatab ,Zrig 6029
}

Gabès, Tunisia

\author{
Saloua Bel Hadj Ali \\ National Engineering School of \\ Gabès , University of Gabès \\ Street Ibn El Khatab ,Zrig 6029
}

Gabès, Tunisia

\author{
Abdelkrim Mohamed Naceur \\ National Engineering School of Gabès, \\ University of Gabès Street Ibn El Khatab ,Zrig 6029 \\ Gabès, Tunisia
}

\begin{abstract}
This paper deals with a Luenberger observer for a class of Takagi Sugeno (TS) descriptor systems. This kind of systems represents many fields as chemical, robotics and electrical systems. An approach with Recursive Least square method is used to study the design of the observer for the Takagi Sugeno descriptor system. An example for non linear descriptor system is given to illustrate the effectiveness of the proposed approach.
\end{abstract}

\section{General Terms \\ Fuzzy Systems.}

\section{Keywords}

Takagi Sugeno, Descriptor System, Observer, Recursive Least Square.

\section{INTRODUCTION}

To describe dynamic behavior of real process non-linear models are widely used in different domain of engineers and scientists. As long as it is difficult to model this kind of system, Takagi Sugeno fuzzy model has proven to be a powerful tool in the analysis and synthesis for control, observer and diagnosis. The TS approach is a powerful technique to model non-linear systems which allows obtaining a good compromise between the precision and the complexity of the model. The interests to use this structure is already well known, most of the available tools of analysis for the linear systems can be extended in the analysis of the non-linear systems, such as observers' synthesis[1], [2], [3], [4].

Since both TS and descriptor formalism are attractive in the field of modeling, a wider class of TS fuzzy systems is described by the descriptor form. In fact, descriptor systems have a tighter representation for a wider class of systems in comparison to traditional state-space models. Descriptor systems represent a more general frame work for non linear systems. A descriptor model is an appropriate model for better describing physical systems than regular ones in many applications. It is well known that descriptor systems (also known as singular systems, implicit systems differentialalgebraic systems, generalized state-space systems) can describe the dynamics of many practical systems such as electrical circuits, economic systems and chemical systems. Most research dealt with measurable premises variables (input and output) for the observer design [5], [6], [7], [8], [9].
In this brief, we will discuss observer design for TS descriptor system using an approach which used Recursive Least square method. This paper is organized as follows. After the system descriptions and definition are presented in section 2, design of Luenberger observer is given in section 3 . Section 4 presents an example to illustrate the effectiveness of the proposed method.Concluding remarks finish this paper.

\section{DESCRIPTION OF THE SYSTEM}

Let us begin by defining the class of the studied systems. A non linear descriptor system is defined, in the continuous case by:

$$
\begin{aligned}
& E(x(t)) \dot{x}(t)=A(x(t)) x(t)+B(x(t)) u(t) \\
& y(t)=C(x(t)) x(t)
\end{aligned}
$$

Where $x(t) \in \square^{n}, u(t) \in \square^{q}$ and $y(t) \in \square^{m}$ represent respectively the state, the input and the output vectors; $A(x(t)), E(x(t)) \in \square^{n \times n}, B(x(t)) \in \square^{n \times m}$ and $C(x(t)) \in \square^{q \times n}$ are non linear matrices functions. For simplicity, we should always consider that $E(x(t))$ is regular for each $x(t) \in \square^{n}$.

Then the descriptor TS system is given as:

$$
\begin{aligned}
& \sum_{\substack{k=1 \\
y(t)=C x(t)}}^{l} v_{k}(\xi(t)) E_{k} \dot{x}(t)=\sum_{i=1}^{r} \mu_{i}(\xi(t))\left(A_{i} x(t)+B_{i} u(t)\right) \\
&
\end{aligned}
$$

Where $\xi$ represent the premise variable; $\mu_{i}(\xi(t))$ and $v_{k}(\xi(t))$ represent respectively the right and the left activating function; $l$ and $r$ is respectively the right and the left number of fuzzy rules; $E_{k}, A_{i} B_{i}$, and $C$ are constant matrices.

$\mu_{i}(\xi(t))=\frac{w_{i}(\xi(t))}{\sum_{i=1}^{r} \mu_{i}(\xi(t))}, w_{i}(\xi(t))=\prod_{i=1}^{g} F_{i j}\left(\xi_{j}(t)\right)$ 
$\mu_{i}(\xi(t))$ has also the same characteristic as $v_{k}(\xi(t))$ and it satisfies:

$$
\begin{gathered}
\mu_{i}(\xi(t))=1 \text { and } \mu_{i}(\xi(t))>0 \text { for } i=1, \ldots, r \\
\quad \text { and } \\
v_{k}(\xi(t))=1 \text { and } v_{k}(\xi(t))>0 \text { for } k=1, \ldots, l
\end{gathered}
$$

\subsection{The sector Non Linearity Method}

In order to illustrate the modeling of the descriptor system by the sector non linearity method we will study an example. The system dynamics is given by the set of differential and algebraic equations. It describes a kind of descriptor system with constant matrix $\mathrm{E}_{\mathrm{k}}=\mathrm{cte}$. The system represents a rolling disk which rolls on surface without slipping .The disk is related to a fixed wall with a non linear spring and a linear damper .The spring has a positive coefficient $k$. The positive parameter $b$ represents the damping coefficient of the damper. The radius of the disk, its inertia and its mass are given respectively by $\mathrm{r}$, $\mathrm{J}$ and $\mathrm{m}$.

The state vector of this model is given by:

$x_{1}(t)$ : The position of the center of this disc

$\mathrm{x}_{2}(\mathrm{t})$ : The translational velocity of the same point.

$\mathrm{x}_{3}(\mathrm{t})$ : The angular velocity of the disc.

$\mathrm{x}_{4}(\mathrm{t})$ : The contact force between the disc and the surface.

$$
\left\{\begin{array}{l}
\dot{x}_{1}=x_{2} \\
\dot{x}_{2}=-\frac{k}{m} \frac{1}{\left(1+x_{1}^{2}\right)} x_{1}-\frac{b}{m} x_{2}-\frac{1}{m} x_{4}+\frac{r}{j} u \\
\dot{x}_{1}=x_{2}-r x_{3} \\
0=-\frac{b}{m} x_{2}-\frac{k}{m} \frac{1}{\left(1+x_{3}^{2}\right)} x_{3}-\frac{r^{2}}{\left(J+\frac{1}{m}\right)} x_{4}-\frac{r}{j} u
\end{array}\right.
$$

\section{Lemma 1:}

Each nonlinear bounded function $f(x)$ can be rewritten as a convex sum of two nonlinear functions [10]:

$$
\begin{aligned}
& \forall x \in[-b, a], f(x) \in\left[f_{\text {min }}, f_{\text {max }}\right] \\
& f(x)=\alpha(x) \times f_{\text {min }}+\beta(x) \times f_{\text {max }}
\end{aligned}
$$

With $\alpha(x) \in[0,1], \beta(x) \in[0,1]$ and $\quad \alpha(x)+\beta(x)=1$.

By using this lemma every non linear term can be simplified and linearized by sector non linearity method.

\subsection{Identification by Recursive Least Square Method}

The difference between this method and the first is the choice of the activating functions. Ever non linearity will be quantified by fuzzy sets. The descriptor TS model is determinate by the modeling of the non linear term. It is assumed that the premise variable belongs to a well defined interval. Then the different locals' sub-system will be obtained by the recursive least square method [11].

\section{DESIGN OF OBSERVER}

For the design of the observer the second method is used. Using the general TS descriptor form (2), it is possible to suppose a TS descriptor observer based on this form:

$$
\begin{aligned}
& \sum_{\substack{k=1 \\
\hat{y}(t)=C \hat{x}(t)}}^{l} v_{k}(\xi(t)) E_{k} \dot{\hat{x}}(t)=\sum_{i=1}^{r} h_{i}(\xi(t))\left[A_{i} \hat{x}(t)+B_{i} u(t)+L_{i}(y(t)-\hat{y}(t))\right] \\
&
\end{aligned}
$$

Where $\hat{x}(t) \in \square^{n}$ and $L_{i}$ represent respectively the estimate state vector and the observer gain matrices.

The fuzzy modeling of system is useful for the design of observer. Indeed, the observer was modeled on the basis of local observers of local linear systems. For the choice of the gain $\mathrm{Li}$ we will use the formula of Bass and Guerra .Then, the observability for descriptor multi-models requires that all the subsystems are observable.

E must be a basis of the $\mathrm{n}$-dimensional vector space:

$$
\operatorname{rank}\left[\begin{array}{c}
E \\
C
\end{array}\right]=n
$$

Each local model of the descriptor TS system has to be observable:

$$
\begin{aligned}
& \forall i=1, \ldots, l . \\
& \operatorname{rank}\left[\begin{array}{c}
s E-A_{i} \\
C
\end{array}\right]=n
\end{aligned}
$$

\section{ILLUSTRATIVE EXAMPLE}

We define a non linear descriptor system defined as:

$$
\left\{\begin{array}{l}
1.5 \dot{x}_{1}=x_{2} \\
\dot{x}_{2}=-2 x_{1}-0.5 x_{2}-\sin x_{1}\left(x_{1}\right)+0.5 u
\end{array}\right.
$$

By supposing that $x_{1} \in[-\Pi / 2+\Pi / 2]$ therefore it will be quantified by fuzzy sets, (see Figure 1). 


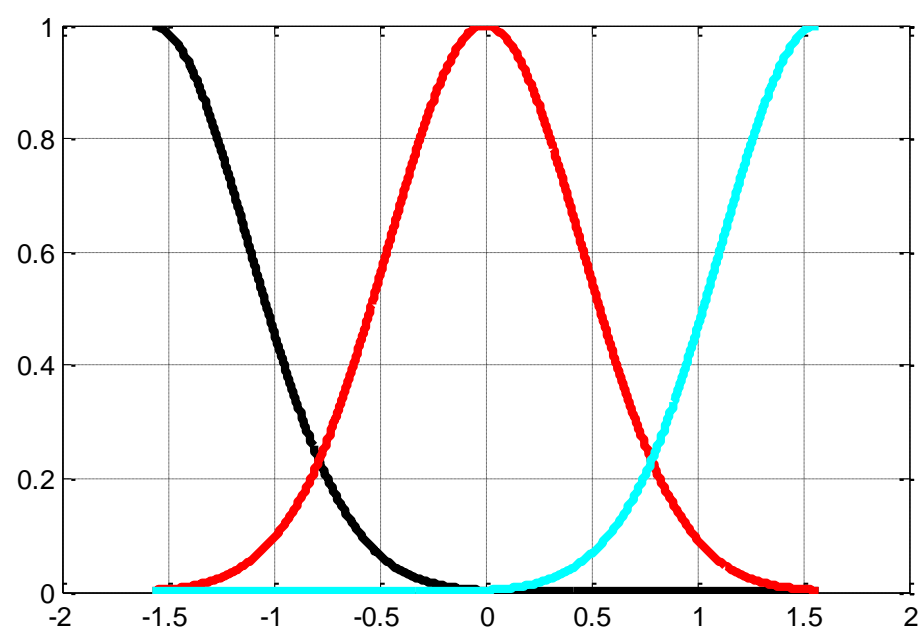

Fig 1: Activating functions

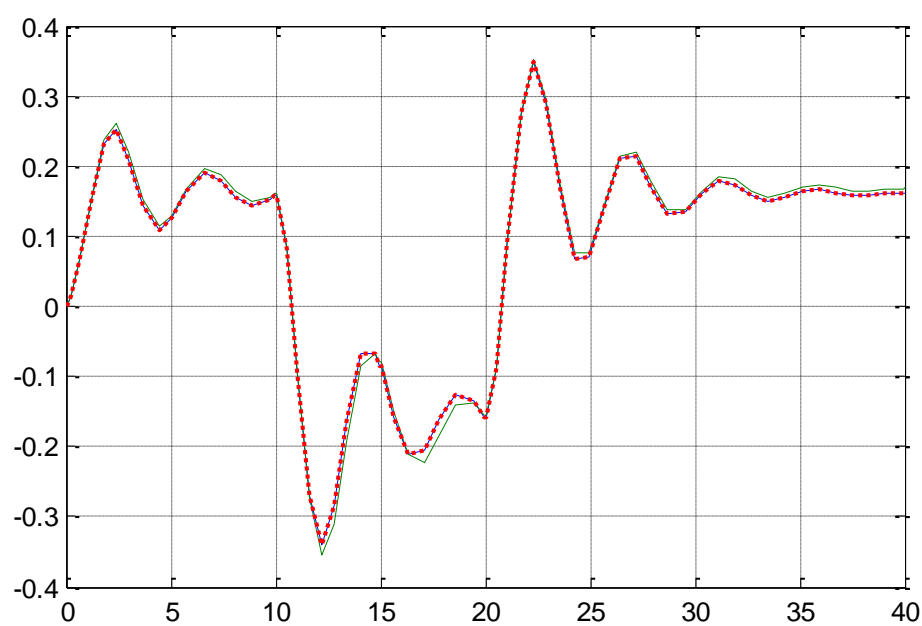

Fig 2: $x_{1}(t)$ of the model and $x_{1}(t)$ estimated

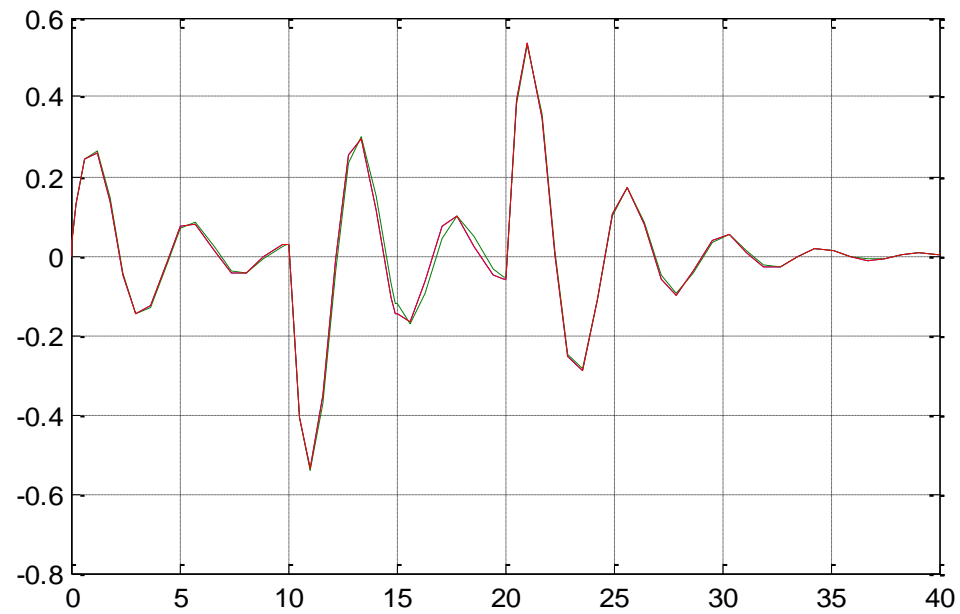

Fig 3: $x_{2}(t)$ of the model and $x_{2}(t)$ estimated 
For computing of gains $\mathrm{L}_{\mathrm{i}}$ the desired performances are characterized by a double pole witch equal to $(-2)$.

The answer of the observer is very close to that of the model. (Figure 2 and Figure3).

\section{CONCLUSION}

The design of Luenberger observer was studied in this paper. The considered systems are modeled in the Takagi Sugeno descriptor structure. A further research should look at the non linearity in the left side of the system.

\section{REFERENCES}

[1] Tanaka, K., Ikeda, T., and Wang, H.O 1998, Fuzzy regulators and fuzzy observers: relaxed stability conditions and lmi-Based designs, IEEE Trans. Fuzzy Syst, vol. 6, pp. 250-265.

[2] Bergsten, P., Palm R. and Driankov, D. 2001, Fuzzy observers, Proc 10th IEEE Internat Conf on Fuzzy Systems, vol. 2, pp.700-703.

[3] Bergsten, P., Palm R. and Driankov, D. 2002, Observers for Takagi-Sugeno fuzzy systems, IEEE Transactions on Systems Man and Cybernetics, pp.114121.

[4] Bergsten, P., Palm, R., and Driankov, D. 2002, Observers for Takagi-Sugeno fuzzy systems, IEEE Transactions on Systems Man and Cybernetics, pp.114121

[5] Lu, G., Wang, D., and Sun, Y. 2005, Observer design for a class of non linear descriptor systems, International
Conference on Control and Automation, Budapest, Hungary.

[6] Kilani, I., Jabri, D., Bel Hadj Ali, S., and Abdelkrim, M.N.2012, Observer design for Takagi-Sugeno descriptor system with lipschitz constraints, International Journal of Instrumentation and Control Systems (IJICS), pp.13-25.

[7] Kilani, I., Jabri, D., Bel Hadj Ali, S., and Abdelkrim, M.N 2013, An Unknown Input Observer for Takagi Sugeno Descriptor System with Unmeasurable Premise Variable, The International Multi-conference on Systems, Signal and Devices SSD.

[8] Kilani, I., Jabri, D., Bel Hadj Ali, S., and Abdelkrim, M.N. 2013, Observer for Takagi-Sugeno Descriptor System Using Two Approaches, The 14th International Conference on Sciences and Techniques of Automatic Control \& Computer Engineering STA.

[9] Ragot, J. 2011, Diagnosis and control using multiple models: application to a biological reactor, Proceedings of the 4th International Symposium on Advanced Control of Industrial Processes, China.

[10] BOUARAR, T.2009, Mise en œuvre de lois de commande pour les modèles flous de type TakagiSugeno, Thèse de doctorat, l'Université de Reims Champagne Ardenne.

[11] Ouakka, H. 2009, Contribution à l'identification et la Commande Floue d'une Classe de Systèmes non Linéaires 2009, Thèse de doctorat, Faculté des sciences Dhar el Mehraz de FES. 